\title{
Current Concepts in the Medical Management of the Pediatric Burn Patient
}

\author{
Carlos Duran $^{1,2,4}$ • Robert L. Sheridan ${ }^{1,3}$
}

Published online: 17 September 2016

(C) Springer International Publishing AG 2016

\begin{abstract}
Purpose of Review Management of the pediatric burn patient involves a careful, multi-faceted approach in order to meet treatment goals. Due to advances in surgical and medical care, mortality and morbidities can be minimized. This chapter seeks to address current advances which allow practitioners to provide excellent systemic care.

Recent Findings Judicious fluid management of children with severe burn injury can improve the respiratory outcome measures of these children. A multi-modal approach to pain and sedation can improve the neurologic status of severely burned children. Summary Children with significant burn injury can have the most favorable outcomes when the principles of skillful critical care are applied. Systemic critical care based on the best evidence available not only improves survival rates but also improves quality of life for these delicate patients.
\end{abstract}

Keywords Burns - Capillary leak · Fluid resuscitation . Hypermetabolic response · Sepsis · Post-traumatic stress disorder

This article is part of the Topical Collection on Pediatric Trauma

Carlos Duran

CDURAN@PARTNERS.ORG

1 Shriners Hospital for Children-Boston, 51 Blossom Street, Boston, MA 02114, USA

2 Division of Pediatric Critical Care, Massachusetts General Hospital, 175 Cambridge Street, Boston, MA 02114, USA

3 Division of Burns and Department of Surgery, Massachusetts General Hospital, 55 Fruit Street, Boston, MA 02144, USA

4 Harvard Medical School, 25 Shattuck, Boston, MA 02115, USA

\section{Introduction}

Every year in the USA, approximately 2 million people are burned. Eighty thousand people are hospitalized. Pediatrics makes up 120,000 emergency room visits a year [1•]. Seventy percent of pediatric admissions are associated with exposure to hot liquids, whereas flame burns are more common in adolescents. Up to $20 \%$ of inpatient cases involve nonaccidental trauma. Advances in the care of the pediatric burn patient over the last decade have led to decreased morbidities and shorter lengths of stay. This chapter focuses on the current medical strategies which enable providers to best care for pediatric burn patients.

\section{Physiology}

Patients with burns less than $10 \%$ of the body surface are often managed in the outpatient arena. Burns larger than this generally require hospital admission. The American Burn Association uses a set of criteria to assist in selecting patients for transfer to dedicated burn units (Table 1). The care for patients with deep partial thickness and full thickness wounds often follows a typical pattern, which is described in Table 2.

At the tissue level, fairly predictable physiologic changes also occur [2]. These changes are summarized in Table 3. The "ebb phase" is a period within a few days of the injury in which there is a relative hypodynamic state and diffuse capillary leak. It is during this period that patients receive fluid resuscitation and if done properly, it can positively affect their progress over the rest of the hospitalization. Overly aggressive fluid resuscitation can lead to a number of morbidities including pulmonary edema and extremity compartment syndrome. The "flow phase" refers to a period of protracted high cardiac output, low systemic vascular resistance, and muscle 
Table 1 The American Burn Association burn center transfer criteria

1. Partial thickness burns greater than $10 \%$ total body surface area.

2. Burns that involve the face, hands, genitalia, perineum, or major joints.

3. Full thickness burns in any age group.

4. Electric burns, including lightning injury.

5. Chemical burns.

6. Inhalation injury.

7. Burn injury in patients with preexisting medical disorders that could complicate management.

8. Any patient with burns and concomitant trauma (such as fractures) in which the burn injury poses the greatest risk of morbidity or mortality. In such cases, if trauma poses a greater immediate risk, the patient may be initially stabilized in a trauma center.

9. Burned children in hospitals without qualified personnel or equipment for the care of children.

10. Burn injury in patients who require special social, emotional, or rehabilitative intervention.

catabolism. This period typically lasts until there is bulk wound closure. Following wound closure, hypermetabolic physiology generally subsides.

\section{Initial Evaluation of Patients with Large Burns}

For all pediatric patients arriving to the ICU with significant burn injuries, providers need to consider the possibility of multiple trauma and incomplete evaluation [3]. The evaluation of these patients should follow the survey format of the American College of Surgeons Committee on Trauma, Advanced Trauma Life Support Course.

The first and most important aspect of the primary survey of pediatric burn patients is evaluation and control of the airway. If there is a significant burn to the face or upper chest, progressive airway edema can develop in the epiglottic and glottic regions over the first few post-injury hours. This is especially true in infants and toddlers. If the patient has hoarseness, stridor, cough, or carbonaceous sputum, intubation is necessary. Intubation can generally be accomplished safely with direct laryngoscopy if the edema is mild. If the patient's airway is already swollen, then it is critical to have flexible bronchoscopy and other surgical airway equipment ready as well as anesthesia personnel at bedside. Once the endotracheal tube has been placed successfully, it must be secured properly. The next key step is to assess the rate and depth of respiration and provide oxygen if necessary. If there is full thickness circumferential burn of the anterolateral torso, early thoracic escharotomy may be necessary. Children involved in house fires are also at significant risk for inhalational injury. Signs of inhalation injury can include ignited clothing, singed nasal hairs, and respiratory distress with and without hypoxemia [4•]. The treatment of inhalational injury includes oxygen therapy, nebulized albuterol, and nebulized epinephrine. In some cases, nebulized heparin is also effective. An evaluation for carbon monoxide toxicity and cyanide poisoning is also necessary when inhalational injury is suspected.

The next most vital aspect of care in the pediatric burn patient is circulation assessment and vascular access. An accurate pulse and blood pressure must be documented to assess for signs of hypovolemic shock. Shock can then be treated according the Society of Critical Care Sepsis guidelines [5]. If there are no signs of shock, then the patient can be started on fluid rates as suggested by the American Burn Life Support guidelines [6]. The suggested fluid rates are listed in Table 4. The perfusion of burned fingertips and toes must also be assessed for early signs of extremity compartment syndrome. If pulses cannot be assessed by Doppler, then escharotomies may be necessary to prevent tissue necrosis. In regards to establishing access, attempts at peripheral intravenous access may be limited by the location of the burn. Thus, if peripheral access cannot be established, then an intraosseous line needs to be established, consistent with PALS guidelines [7]. In some cases, central cannulation may be considered from the onset if the size and depth of the burn is severe.

\section{Fluid Resuscitation}

Fluid resuscitation of pediatric burn patients has long been a topic of much discussion in critical care circles. Several burn resuscitation formulas, based on the size of the burn using the Lund-Browder Chart (Fig. 1), have been developed to counteract the diffuse capillary leak that occurs during the "ebb" phase. While not all the formulas are the same, the goals of
Table 2 Phases of burn care for large burns (>20\% TBSA)

\begin{tabular}{lll}
\hline Phase & Time course & Goals \\
\hline Initial evaluation and resuscitation & $0-72 \mathrm{~h}$ & $\begin{array}{c}\text { Complete assessment of injuries and judicious } \\
\text { fluid resuscitation }\end{array}$ \\
$\begin{array}{l}\text { Initial wound excision and } \\
\text { biologic closure }\end{array}$ & Days 1-7 & $\begin{array}{c}\text { Accurate wound assessment with minimal wound } \\
\text { excision; typically allograft placement } \\
\text { Definitive wound closure }\end{array}$ \\
$\begin{array}{l}\text { Rehabilitation } \\
\text { Days 7-30 }\end{array}$ & Day 1-discharge & $\begin{array}{c}\text { Maintain range of motion; strengthening } \\
\text { and emotional recovery }\end{array}$ \\
\end{tabular}


Table 3 Major physiologic changes in burn patients

\begin{tabular}{|c|c|c|}
\hline Time period & Physiology & Treatment plan \\
\hline $\begin{array}{l}\text { Day } 0-3 \text { : resuscitation; } \\
\text { "ebb" phase }\end{array}$ & $\begin{array}{l}\text { Diffuse capillary leak; hypodynamic } \\
\text { circulation }\end{array}$ & $\begin{array}{l}\text { Judicious fluid resuscitation } \\
\text { needed }\end{array}$ \\
\hline $\begin{array}{l}\text { Day 3-bulk wound closure; } \\
\text { "flow" phase }\end{array}$ & Hyperdynamic, hypermetabolic state & $\begin{array}{l}\text { Wound closure; infection control; } \\
\text { nutrition support }\end{array}$ \\
\hline Bulk wound closure -2 years & Tapering of the hypermetabolic state & $\begin{array}{l}\text { Continued nutritional support; } \\
\text { rehabilitation }\end{array}$ \\
\hline
\end{tabular}

resuscitation are similar. These include a child with a normal mental status, adequate perfusion and blood pressure, and good urine output. The child should also have a normal base deficit and a normal lactate level. With these goals in mind, the modified Brooke formula is widely accepted as a good starting point for fluid resuscitation [8•]. The fluid resuscitation then is titrated hourly according to a urine output of $1-2 \mathrm{ml} / \mathrm{kg} / \mathrm{h}$, and this typically relies on frequent monitoring of the patient. The use of colloid in fluid resuscitation continues to be debated, and a number of current providers advocate for the use of colloid in the first $24 \mathrm{~h}$, depending on the size of the burn $[9,10]$.

\section{System by System Management}

A system by system approach to the pediatric burn patient provides the framework for delivering comprehensive care. During the period of diffuse capillary leak following a major burn, the cardiovascular system may be the focus but pain and anxiety issues are a constant and will need attention well after the bulk of wound closure has been completed. The following section describes how current practice has evolved.

\section{Neurologic System}

Pain and anxiety are critical issues for the pediatric burn patient, and management of these issues begins with the first wound evaluation happening in the urgent care center or emergency room. Typically, an opioid, such as morphine or fentanyl, will be administered to allow for thorough cleaning of the affected area. In years prior, it was common for the opioid to be delivered via the intravenous route. However, this would involve placing a peripheral
IV, and this is not necessarily a trivial task in a toddler. Oral morphine or fentanyl "lollipops" were an alternative but would often take 30-45 min to take effect. More recently, agents such as fentanyl can be given via the intranasal route and have equivalent pain control as compared to oral morphine for dressing changes [11]. This practice has become commonplace in a number of referring hospitals when peripheral IV access is not established. In terms of anxiety management, fast-acting benzodiazepines such as lorazepam and midazolam remain the treatment of choice. Midazolam is the preferred agent in that it has a shorter duration of action and can be delivered by the intranasal route as well [12].

After transfer to a dedicated burn unit, the child can be fitted with an individualized pain management plan. The plan can then be titrated according to pain scores. In addition, the pain management plan can be delineated according to the aspects of background pain, procedural pain, and postoperative pain. In regards to background pain, non-opioids (e.g., acetaminophen, ibuprofen) and low-potency opioids such as oxycodone are adequate for wounds $<10 \%$ total body surface area (TBSA). Anxiety can be managed with lorazepam on an as needed basis. For burns injuries $>20 \%$, typically scheduled, intermittent doses of IV morphine are necessary. Morphine has an advantage over fentanyl in this setting in that morphine has a longer duration of action and intermittent dosing of fentanyl can be associated with chest wall rigidity [13]. Scheduled lorazepam is generally needed for anxiolysis at this point. For wounds $20-40 \%$, continuous morphine is recommended for pain control and continuous midazolam for anxiolysis. Again, morphine is preferable in this situation over continuous fentanyl, in that the later is associated with a greater risk of tolerance [14]. While continuous midazolam is associated with greater tolerance, continuous lorazepam for greater than $72 \mathrm{~h}$ is associated with the formation of
Table 4 Pediatric burn caloric formula

\begin{tabular}{lll}
\hline Age group & Maintenance needs & Burn wound needs \\
\hline Infants $(0-12$ months $)$ & $2100 \mathrm{kcal} \times \mathrm{m}^{2}$ & $\left(1000 \mathrm{kcal} \times \mathrm{m}^{2} \times \%\right.$ TBSA $) / 100$ \\
Children $(1-12$ years $)$ & $1800 \mathrm{kcal} \times \mathrm{m}^{2}$ & $\left(1300 \mathrm{kcal} \times \mathrm{m}^{2} \times \%\right.$ TBSA $) / 100$ \\
Adolescents $(12-18$ years $)$ & $1500 \mathrm{kcal} \times \mathrm{m}^{2}$ & $\left(1500 \mathrm{kcal} \times \mathrm{m}^{2} \times \%\right.$ TBSA $) / 100$ \\
\hline
\end{tabular}

Jeschke, M et al. Sabiston Textbook of Surgery. 2012 


\begin{tabular}{|c|c|c|c|c|c|c|c|c|c|c|}
\hline Area & $\begin{array}{l}\text { Birth to } \\
1 \text { year }\end{array}$ & $\begin{array}{l}1 \text { to } 4 \\
\text { years }\end{array}$ & $\begin{array}{l}5 \text { to } 9 \\
\text { years }\end{array}$ & $\begin{array}{l}10 \text { to } 14 \\
\text { years }\end{array}$ & $\begin{array}{l}15 \\
\text { years }\end{array}$ & Adult & $2 n d^{*}$ & $3 d^{*}$ & TBSA & \\
\hline Head & 19 & 17 & 13 & 11 & 9 & 7 & & & & \\
\hline Neck & 2 & 2 & 2 & 2 & 2 & 2 & & & & \\
\hline Anterior trunk & 13 & 13 & 13 & 13 & 13 & 13 & & & & \\
\hline Posterior trunk & 13 & 13 & 13 & 13 & 13 & 13 & & & & \\
\hline Right buttock & 2.5 & 2.5 & 2.5 & 2.5 & 2.5 & 2.5 & & & & \\
\hline Left buttock & 2.5 & 2.5 & 2.5 & 2.5 & 2.5 & 2.5 & & & & \\
\hline Genitalia & 1 & 1 & 1 & 1 & 1 & 1 & & & & \\
\hline Right upper arm & 4 & 4 & 4 & 4 & 4 & 4 & & & & \\
\hline Left upper arm & 4 & 4 & 4 & 4 & 4 & 4 & & & & \\
\hline Right lower arm & 3 & 3 & 3 & 3 & 3 & 3 & & & & \\
\hline Left lower arm & 3 & 3 & 3 & 3 & 3 & 3 & & & & \\
\hline Right hand & 2.5 & 2.5 & 2.5 & 2.5 & 2.5 & 2.5 & & & & \\
\hline Left hand & 2.5 & 2.5 & 2.5 & 2.5 & 2.5 & 2.5 & & & & \\
\hline Right thigh & 5.5 & 6.5 & 8 & 8.5 & 9 & 9.5 & & & & \\
\hline Left thigh & 5.5 & 6.5 & 8 & 8.5 & 9 & 9.5 & & & & \\
\hline Right leg & 5 & 5 & 5.5 & 6 & 6.5 & 7 & & & & \\
\hline Left leg & 5 & 5 & 5.5 & 6 & 6.5 & 7 & & & & \\
\hline Right foot & 3.5 & 3.5 & 3.5 & 3.5 & 3.5 & 3.5 & & & & \\
\hline Left foot & 3.5 & 3.5 & 3.5 & 3.5 & 3.5 & 3.5 & & & & \\
\hline & & & & & & \begin{tabular}{|l|} 
Total: \\
\end{tabular} & & & & \\
\hline
\end{tabular}

*-Second-degree burns are now more often designated as superficial partial-thickness or deep partial-thickness burns, and third-degree burns are designated as full-thickness burns.

Fig. 1 Lund-Browder Bum Estimate Chart (adapted with permission from Mertens DM, Jenkins ME, Warden GD. Outpatient burn management. Nurs Clin North Am 1997;32:343-64, and Lund C,

unfavorable metabolites, and thus the former is preferred. For wounds $>40 \%$, dexmedetomidine, an $\alpha$-2 agonist, is added to the regimen of continuous morphine and midazolam. The early use of dexmedetomidine is helpful in preventing the escalation of morphine and midazolam doses via continuous infusions [15]. Finally, once a patient has been on continuous narcotics or benzodiazepines for greater than 5 days, he is at greater risk for withdrawal. Thus, these patients are scored with the Withdrawal Assessment Tool (WAT-1) to allow for successful weaning of the sedatives.

Procedural pain tends to be more intense and shorter in duration than background pain. For this reason, intravenous short-acting narcotics such as fentanyl or narcotics with sedative effects such as morphine are useful for pain control. Intravenous midazolam is effective as an anxiolytic. When a child is considered to be at risk for non-compliance or the wound area is large, ketamine is treatment of choice for providing analgesia and amnesia via its NMDA receptor activity.
Browder N. The estimation of areas of burns. Surg Gynecol Obstet 1944;79:352-8.)

Post-operative pain is also different from background pain in that there are increased analgesic needs. This is especially true following autografting, in which the greatest source of pain is the donor site area which has been harvested. For small areas of autograft, intermittent intravenous morphine and ativan will suffice. When larger areas have been harvested, continuous infusions of morphine, midazolam, and dexmedetomidine for 1-3 days following surgery are ideal [16].

\section{Cardiovascular System}

As described earlier, the trauma of a large burn induces microvascular changes which lead to a variety of cardiovascular alterations. During the "ebb phase," there is loss of plasma volume, increased peripheral vasculature resistance, and subsequent decreased cardiac output immediately after injury. 
Cardiac output is depressed because of decreased blood volume and increased blood viscosity, as well as decreased cardiac contractility [17]. A tailored fluid resuscitation plan is necessary to navigate the patient safely through this phase. In addition, the patient will benefit from central venous cannulation for reliable access. An arterial line provides continuous blood pressure monitoring and can also be used for multiple lab draws. Monitoring central venous pressure and urine output are helpful in assessing the success of an individualized fluid plan. Following the "ebb" phase, the pediatric burn patient then enters a hyperdynamic, hypermetabolic state of muscle catabolism. During this phase, the patient can experience a marked tachycardia (upwards of $150 \%$ predicted). This often requires treatment with propranolol, a non-selective $\beta$ blocker, which decreases chrontropy. Propranolol also has other metabolic effects which will be discussed in the nutritional support section.

\section{Pulmonary System}

Given the importance of this system, much has already been described in the "Initial Evaluation of Patients with Large Burns" section above. However, lower airway disease and systemic toxicity (i.e., carbon monoxide poisoning) merit further discussion. Injury to the upper airway can occur with any burn to the face but inhalational injury is the main cause of lower airway injury and systemic toxicity. Lower airway injury is characterized by oxidative stress, excess mucus secretion, bronchiolar obstruction, and ventilation-perfusion mismatch. In mild cases, this can be treated with nasal cannula $\mathrm{O}_{2}$ or high flow nasal cannula (HFNC). When these oxygen therapies are insufficient, continuous positive airway pressure (CPAP) and bilevel positive airway pressure $(\mathrm{BiPaP})$ can be used. When these non-invasive methods are not enough, mechanical ventilation is employed. Low-tidal volume ventilation with adequate positive end-expiratory pressure (PEEP) is then used to maximize oxygenation and decrease the risk of barotrauma. Current treatment strategies such a conservative fluid management plan and a daily lightening of sedation are helpful in reducing the duration of mechanical ventilation and decreasing the risk of acute respiratory distress syndrome (ARDS) [18•].

Regarding systemic toxicity, inhalation injury can cause carbon monoxide poisoning and cyanide poisoning. Carbon monoxide (CO) binds to hemoglobin much more avidly than does oxygen, forming carboxyhemoglobin $(\mathrm{COHb})$. $\mathrm{COHb}$ prevents the normal capacity of hemoglobin to deliver oxygen to the tissues. Carbon monoxide also binds to the mitochondrial electron transport chain leading to oxidative stress. Symptoms of CO toxicity include headache, nausea, and, in severe cases, coma. Treatment of $\mathrm{CO}$ poisoning is $100 \%$ oxygen until the $\mathrm{COHb}$ is less than $5 \%$ [8•]. This can also be accomplished by providing the patient with hyperbaric oxygen. Cyanide also interferes with the mitochondrial electron transport chain and leads to lactic acidosis which is out of proportion to the burn size. It can cause cardiovascular collapse and unconsciousness. Treatment of cyanide poisoning is high-dose hydroxocobalamin (Cyanokit).

\section{Gastrointestinal System}

The initial goal of this section is to protect the stomach from mucosal injury and avoid severe intraabdominal complications such as abdominal compartment syndrome and nonocclusive mesenteric ischemia. With regards to mucosal injury, antacids, $\mathrm{H}_{2}$-blockers, and proton-pump inhibitors provide adequate protection to prevent stress ulcers. They are often instituted on arrival to the burn unit, especially when there is a large burn or the patient will remain NPO. Abdominal compartment syndrome can best be avoided by careful initial fluid resuscitation and monitoring of abdominal girth and urine output [19]. Nonocclusive mesenteric ischemia presents as necrosis of various portions of the small and/or large intestine. It typically occurs later than abdominal compartment syndrome and may be related to the use of vasopressors and massive burn wound excision.

While a pediatric patient with a significant burn may be protected from stress ulcers, bowel function often is still impaired and can also be a consequence of narcotics used for pain control. Typically, patients will present with constipation, and this needs to be addressed once enteral nutrition has been established. Agents such as docusate and senna are often scheduled as prophylaxis. If needed, bisacodyl suppositories and Miralax can also be used to produce stool. The pancreas and liver also can be become inflamed when there is greater than $20 \%$ TBSA burn injury. Acalculous cholecystitis can also be an obstructive cause of hyperbilirubinemia in children with burns. Treatment typically involves gallbladder drainage.

\section{Nutritional Support}

The hypermetabolic response to a burn injury occurs during the "flow phase." Increases in oxygen consumption, metabolic rate, urinary nitrogen excretion, lipolysis, and weight loss are directly proportional to the size of the burn. The response can be as much as $200 \%$ of the normal metabolic rate and only returns to normal when there is complete closure of wound. Due to this high metabolic rate, energy requirements are enormous and are met by mobilizing carbohydrate, fat, 
and protein stores. These energy stores are quickly depleted, leading to malnutrition and muscle tissue loss. The malnutrition leads to delayed and abnormal wound healing and decreased immune function. Delivery of exogenous nutritional support can help combat malnutrition. Table 4 shows an example of predicted caloric needs in severely burned children. In addition, the nutritional support optimally has a composition of $1-2 \mathrm{~g} / \mathrm{kg} /$ day of protein. This amount of protein intake meets the synthetic needs of the patient. Carbohydrates also provide an excellent source of nonprotein calories. They stimulate endogenous insulin production, which has an anabolic effect on muscle and burn wounds. Frequently, children are fitted with a nasogastric tube in order to meet the increased caloric demands of wound healing. However, since some patients may require moderate sedation with ketamine for dressing changes and other minor procedures, a post-pyloric tube may be advantageous. With a post-pyloric feeding tube in place, the patient can continue to receive enteral nutrition prior to the procedure and also avoid additional exposure to general anesthesia [20]. Total parenteral nutrition is generally reserved for patients who cannot tolerate enteral feeds. Additionally, the nutritional progress of the patient can be tracked by following CRP and pre-albumin levels serially. In the child who is receiving adequate nutrition, the CRP will trend lower and pre-albumin level higher.

Oxandolone, a testosterone analogue that has $5 \%$ of the virilizing androgenic effects of testosterone, improves muscle protein catabolism, reduces weight loss, and increases donor site wound healing. It is typically started early in burn injury cases with $>20 \%$ TBSA involvement. While a number of studies have shown its effectiveness in maintaining lean body mass and decreasing the hypermetabolic state of burn injury, there is no consensus on how long the agent should be continued [21•]. In some centers, oxandrolone is continued in the outpatient phase of care, 6,9 , and even 12 months post initial injury. The main concern in long-term use of oxandrolone is the potential for liver dysfunction. Thus, patients need to be periodically monitored for this complication.

Propranolol, a non-selective $\beta$-blocker, is another agent used in acute burn management to decrease the hypermetabolic state. Its main action is $15-20 \%$ reduction in the resting heart rate of a pediatric patient with $>20 \%$ TBSA burn injury. Via its metabolic effect on the liver, it also increases protein synthesis and reduces hepatic stores. Overall, this leads to an increase in lean body mass, and like oxandrolone, it is started early in the inpatient course. However, there is consensus on how long propranolol should be continued.

Aside from hypercaloric enteral feeding regimens and the pharmacologic agents described above, it is worth noting that temperature control can also affect the insensible losses of a pediatric burn patient. In patients with $>35 \%$ TBSA burn, raising the ambient temperature from 25 to $33{ }^{\circ} \mathrm{C}$ can decrease the resting energy expenditure by $30 \%$.

\section{Hematologic System}

The pediatric burn patient with a burn $>20 \%$ TBSA is at risk for anemia and disseminated intravascular coagulation (DIC). Anemia can occur in the setting of an aggressive fluid resuscitation regimen, but is also commonly seen during the excision or autografting phase of burn care. Most units will use a hemoglobin of $7 \mathrm{~g} / \mathrm{dl}$ as a threshold for transfusion, unless there is active brisk bleeding or the patient is clinically unstable in another way. As far as DIC, it is seen in larger burns in which sepsis is a consideration. Prolonged PT, prolonged PTT, low fibrinogen, and elevated D-dimer are markers of disease. Treatment often involves the replacement of plasma products.

\section{Infectious Disease System}

Infection in the pediatric burn patient is a major cause of morbidity and remains a focus of acute burn care. For smaller partial thickness burns, topical agents such as bacitracin (or bactroban for cases colonized with MRSA) are sufficient. If these wounds have more drainage or have a cellulitic appearance, then a course of oral antibiotics with good streptococcal or staphylococcal coverage (e.g., cephalexin) is appropriate. When the wounds are full thickness, there is greater risk for sepsis, and this risk persists until there is excision of the necrotic tissue and closure of the wounds. The approach to antibiotic prophylaxis and treatment changes after 7-10 days when the prominent bacterial wound colonization transitions from grampositive organisms to gram-negative ones such as Pseudomonas, Serratia, and Acintobacter. Consequently, when there is a concern for wound infection prior to excision, broad spectrum coverage with vancomycin and cefepime is common. When there is the threat for multi-drug resistant organisms, meropenem and/or colistin can be added for better protection. These antimicrobial agents are also in the peri-operative arena when excision and grafting is thought to cause to transient bacteremia. In some cases when the wounds look more infected, the antibiotics can be continued for 48-72 $\mathrm{h}$ post-excision or post-grafting. Aside from the systemic agents described, several topic agents such silver nitrate and sulfamylon can be used for local infection control in the pre- and post-excision phases of care [22]. Fungal coverage for wounds can also be accomplished by adding amphotericin to the topical agents or using systemic agents such as micafungin and amphotericin B.

Fever in the pediatric burn patient is common post-excision and wound closure and is not necessarily a sign of infection [23]. Nonetheless, when there is a high fever (e.g., $T>39^{\circ} \mathrm{C}$ ) greater than $24 \mathrm{~h}$ post-op, clinicians need to perform a thorough physical examination to look for infection. If the patient has a central venous catheter, a peripheral blood culture is recommended so catheter colonization is not misconstrued as bacteremia. Sputum culture is recommended in the 
intubated patient, and urine culture should also be obtained in the patients with a Foley. If the patient appears unstable, empiric broad-spectrum antibiotic coverage is warranted until culture data returns. If no infectious etiology can be identified and the patient appears clinically well, antibiotics can be discontinued. In addition, there should be daily assessment of the need for the central venous catheter and Foley catheter, in accordance with the quality and safety benchmarks of all pediatric inpatient units.

\section{Psychiatric System}

The mental state of the pediatric burn patient is fragile and from the time of the first dressing, there is a high risk of anxiety and fear. Midazolam in the intranasal and oral form are excellent for the toddler with no IV access who needs a dressing change. But if anxiolytic is not used or the patient has had multiple dressings with poor pain control, then the patient can develop long-standing anxiety. In this situation, a trained psychiatrist is needed to assess the patient and make recommendations. Respiridone, an antipsychotic medication thought to act through dopamine type 2 and serotonin type 2 receptor antagonism, has found a role in the inpatient treatment of anxiety in pediatric burn patients [24]. Its use needs to be monitored by periodically checking the QTc interval on an EKG. Additionally, sertraline, a selective serotonin reuptake inhibitor, can be used to address the depressive symptoms of select pediatric burn patients. Child life therapy and daily assessment of anxiety and depression symptoms can lead to a successful emotional state for the child.

Delirium is another issue which may arise in the pediatric burn patient. It is characterized by fluctuating mental status, inattention, and an altered level of consciousness. Risk factors for delirium include multi-system disease and prolonged use of the very analgesics and sedatives used in the care of pediatric patients with large burn injuries. While delirium often presents with a patient who is loud and uncontrollable, it can also be the picture of a child who does interact with his environment and thus, it is thought to be under-diagnosed. The Delirium Rating Scale has been used widely to diagnose and rate the severity of delirium in adults. More recently, it has also been validated as an effective screening tool in children [25]. Other screening tools such as the Cornell Assessment of Pediatric Delirium (CAP-D) and the Pediatric Confusion Assessment Method for the Intensive Care Unit (p-CAMICU) have gained popularity as well. Once delirium has been diagnosed, it can be treated with non-pharmacologic measures such as daily awakening from continuous sedation agents and improved sleep. Haloperidol and atypical antipsychotic medications are the common pharmacologic treatments. Other agents, such as clonidine and dexmedetomidine, both $\alpha$ agonists have been used with good effect.

\section{Special Considerations}

\section{Frostbite}

Soft tissue necrosis from cold injury, also known as frostbite, can present in the setting of systemic hypothermia. Before it can be addressed, the core temperature must first be corrected to a minimum temperature of $34^{\circ} \mathrm{C}$. After the patient has been removed from the cold and admitted to an intensive care unit, rapid rewarming of the involved area is the single most effective therapy for frostbite and is best accomplished in circulating water at $37-39^{\circ} \mathrm{C}$ [26]. Warming should be continued for 15-30 min or until thawing is complete. During the thawing process, it is not uncommon for patients to experience reperfusion pain, and this pain needs to be managed in order for the thawing process to be completed. After thawing is complete, the affected area should be elevated and nonadherent dressings applied. In cases in which affected areas do not reperfuse and ischemia time was less than $24 \mathrm{~h}$ prior, intravenous or intra-arterial thrombolysis with tissue plasminogen activator (tPA) has been shown to decrease amputation rates [27]. tPA is generally delivered as a bolus followed by a continuous infusion, along with a heparin infusion. The efficacy of this treatment can be assessed with angiograms. Over the next 4 8 weeks, nonviable tissue will declare itself, and surgical debridement and reconstruction can then be carried out.

\section{Nonaccidental Trauma}

Nonaccidental trauma or neglect has been reported in up to $20 \%$ of pediatric burns. The history and physical exam typically lend important clues to the diagnosis. Documentation of conflicting reports from involved caretakers, delay in seeking treatment, and prior injuries should raise a provider's concerns. From a physical exam standpoint, with regards to scald wounds in particular, uniform burn depth, absence of splash marks, sharply defined wound margins, flexor sparing, and stocking and glove burn patterns are suspicious for forced immersion. Dorsal location of contact burns of the hand and burns in the clear shape of a hot object (i.e., clothing iron) are also worrisome [28]. Any case which raises a suspicion for nonaccidental trauma should be filed with the appropriate child-protection agency.

\section{Conclusions}

Pediatric burn injury presents a multisystemic disease which requires a step-wise approach to address various issue concurrently. Careful consideration of the nature and depth of the injury is critical to providing optimal initial fluid resuscitation and psychological support. Advances in the pain and sedation management of these children may alleviate the burden of 
post-traumatic stress disorder. Early identification of infection and sepsis should also provide better outcomes for the pediatric burn patient.

\section{Compliance with Ethical Standards}

Conflict of Interest Drs. Sheridan and Duran declare no conflicts of interest relevant to this manuscript.

Human and Animal Rights and Informed Consent This article does not contain any studies with human or animal subjects performed by any of the authors.

\section{References}

Papers of particular interest, published recently, have been highlighted as:

- Of importance

1. Sheridan, RL. Critical Care of the Burn Patient. In Textbook of Critical Care, 7th edition. 2016 (in press). Dr. Sheridan is an international leader on the management of burns and especially as it pertains to critical care and children. The topics discussed in this work form the background of core knowledge regarding burn injury.

2. Sheridan RL, Tompkins RG. What's new in burns and metabolism. J Am Coll Surg. 2004;198:243-63.

3. Lindford AJ, Lamyman MJ, Lim P. Review of the emergency management of severe burns (EMSB) course. Burns. 2006:32:391.

4. Dries DJ, Endorf FW. Inhalation injury: epidemiology, pathology, treatment strategies. Scand J Trauma Resusc Emerg Med. 2013; 2131. Dr. Dries' work on inhalation injury is outstanding in understanding the many facets of this complex injury.

5. Carcillo JA et al. Clinical practice parameters for hemodynamic support of pediatric and neonatal septic shock: 2007 update from the American College of Critical Care Medicine. Crit Care Med. 2009;37(2):666-88.

6. American Burn Association. Advanced Burn Life Support Guidelines. 2007.

7. American Heart Association. Pediatric Advanced Life Support Guidelines. 2015.

8. Cancio LC. Initial assessment and fluid resuscitation of burn patients. Surg Clin North Am. 2014;94:741-54. Dr. Cancio's work in the Surgical Clinics of North America is excellent in describing the controversies and evidence regarding fluid resuscitation in burn injury.

9. Atiyeh BS, Dibo SA, Ibrahim AE, et al. Acute burn resuscitation and fluid creep: it is time for colloid rehabilitation. Ann Burns Fire Disasters. 2012;25:59-65.

10. Lawrence A, Faraklas I, Watkins $\mathrm{H}$, et al. Colloid administration normalizes resuscitation ratio and ameliorates "fluid creep.". J Burn Care Res. 2010;31:40-7.
11. Borland ML, Bergesio R, et al. Intranasal fentanyl is an equivalent analgesic to oral morphine in paediatric burns patients for dressing changes: a randomised double blind crossover study. Burns. 2005;31(7):831-7.

12. Hansen SL, Voigt DW, Paul CN. A retrospective study on the effectiveness of intranasal midazolam in pediatric burn patients. $\mathrm{J}$ Burn Care Rehabil. 2001;22(1):6-8.

13. Gandhi M, Thomson C, et al. Management of Pain in Children with Burns. Int J Pediatr. 2010; 1-9.

14. Anand KJS, Wilson DF, Berger J, et al. Tolerance and withdrawal from prolonged opioid use in critically ill children. Pediatrics. 2010;125:e1208-25.

15. Belgrade M, Hall S. Dexmedetomidine infusion for the management of opioid-induced hyperalgesia. Pain Med. 2010;11(12): 1819-26.

16. Walker J, Maccallum M, et al. Sedation using dexmedetomidine in paediatric burn patients. J Burn Care Res. 2006;27(2):206-10.

17. Abu-Sittah GS, Sarhane KA, et al. Cardiovascular dysfunction in burns: review of the literature. Ann Burns Fire Disasters. 2012;25(1):26-37.

18. Ingelse SA, Wosten-van Asperen RM, et al. Pediatric acute respiratory distress syndrome: fluid management in the PICU. Front Peds. 2016;4:1-9. This work highlights the importance of fluid management in pediatric acute lung injury. This is especially relevant considering the fluid requirements and shifts which occur in the first 72 hours of a child with a significant burn injury.

19. Streit S, Hebra A. Abdominal compartment syndrome in a three year old child following a severe burn injury. J Ped Surg Case Rep. 2013;1(7):177-9.

20. Capriati T, Cardile S, et al. Clinical management of post-pyloric enteral feeding in children. Exp Rev Gastro Hepat. 2015;9(7): 929-41.

21. Porro LJ, Herndon DN, et al. Five-year outcomes after oxandrolone administration in severely burned children: a randomized clinical trial of safety and efficacy. J Am Coll Surg. 2012;214(4):489-504.

This paper documents the effect of positive effect that oxandrolone can have on the metabolism of children with severe burns.

22. Palmieri TL, Greenhalgh DG. Topical treatment of pediatric patients with burns: a practical guide. Am J Clin Dermatol. 2002;3(8):529-34.

23. Parish RA, Novack AH, Heimbach DM, et al. Fever as a predictor of infection in burned children. J Trauma. 1987;27:69-71.

24. Meighen KG, Hines LA, Lagges AM. Risperidone treatment of preschool children with thermal burns and acute stress disorder. J Child Adol Psychopharm. 2007;17(2):223-32.

25. Turkel SB, Braslow K, Tavare CJ, Trzepacz PT. The delirium rating scale in children and adolescents. Psychosomatics. 2003;44(2): 126-9.

26. Britt LD, Dascombe WH, Rodriguez A. New horizons in management of hypothermia and frostbite injury. Surg Clin North Am. 1991;71(2):345-70.

27. Ibrahim AE, Goverman J, Sarhane KA, Donofrio J, Walker TG, Fagan SP. The emerging role of tissue plasminogen activator in the management of severe frostbite. J Burn Care Res. 2015;36(2):e62-6.

28. Andronicus M, Oates RK, et al. Non-accidental burns in children. Burns. 1998;24(6):552-8. 